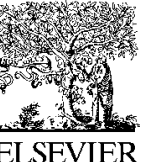

ELSEVIER

\title{
Oxidative phosphorylation inducers \\ feature fight pathological angiogenesis
}

Q2 M.Pilar Bayona-Bafaluy 1,2,3, pbayona@unizar.es, Olivia Esteban ${ }^{2,4}$, Javier Ascaso ${ }^{2,4}$, Julio Montoya ${ }^{1,2,3}$ and Eduardo Ruiz-Pesini ${ }^{1,2,3,5}$, eduruiz@unizar.es

Pathological mutations in subunits of the oxidative phosphorylation (OXPHOS) system, or inhibitors of this biochemical pathway, increase the production of vascular endothelial growth factor (VEGF) and pathological angiogenesis. In many angiogenesis-related diseases, such as retinal, rheumatoid diseases, or cancer, OXPHOS dysfunction can be found. Thus, enhancing OXPHOS might be a promising therapeutic approach for pathologic angiogenesis.

\section{Introduction}

The neovascular form of age-related macular degeneration (nAMD) is a disorder that affects the macular region of the retina and causes progressive loss of central vision. Age is the main risk factor, with nearly all nAMD cases occurring in patients over 60 years old. The disease is characterized by choroidal neovascularization, and intravitreal therapy with ranibizumab, an inhibitor of all VEGFA isoforms, is highly effective [1]. However, a meta-analysis of 1 -year results pointed out that a saturation effect of treatment occurred following an optimal number of 6.87.2 intravitreal ranibizumab injections per year [2]. If this is the case, the increased injection number might reflect refractory cases that respond poorly to ranibizumab. In patients with nAMD, the mitochondrial DNA (mtDNA) genetic background or haplogroup also modifies the response to intravitreal ranibizumab therapy [3]. At 4 months, in all haplogroups, best corrected visual acuity and central foveal thickness (CFT) values were higher and lower than basal values, respectively. There were no differences among genetic backgrounds. However, at 12 months, CFT values of patients from the HV and $U$ haplogroups remained lower than basal values, whereas individuals from the JT haplogroup showed similar CFT values to those before treatment. Moreover, individuals from the $U$ haplogroup showed 12-month CFT values that were significantly lower than those of JT haplogroup. However, individuals from the JT haplogroup received a higher mean number of intravitreal injections (7.1) compared with the other haplogroups. Therefore, other approaches to treating $\mathrm{nAMD}$ should be looked for.

By contrast, mtDNA haplogroups result in groups of phylogenetically related mtDNA genotypes. Particular combinations of mtDNA population polymorphisms define mtDNA haplogroups. mtDNA only encodes OXPHOS system subunits: seven from respiratory complex I (Cl); one from CIII; three from CIV; and two from ATP synthase. It also encodes RNAs required for their expression. The 80 remaining OXPHOS subunits are encoded by nuclear DNA (nDNA). Thus, the mtDNA haplogroup-defining genetic variants might affect OXPHOS function.

Therefore, evidence highlighting the association between mtDNA haplogroups and the clinical results of ranibizumab therapy in nAMD could be the result of a relationship between OXPHOS capacity and angiogenesis.

\section{OXPHOS mutations and inhibitors increase VEGF production and pathological angiogenesis nDNA mutations}

Mutations in nDNA-encoded CII subunits result in decreased CII activity, increased VEGF mRNA expression, and stimulation of the angiogenic pathway in paraganglioma and pheochromocytoma tumors $[4,5]$. Similarly, mutations in the nDNA-encoded CIII subunit UQCRB also decreased OXPHOS function and increased VEGF mRNA and protein expression in UQCRB mutant transfected human embryonic kidney HEK293 cells [6].

Furthermore, vascular proliferation is a common feature of mitochondrial defects in 
different tissues, notably in the central nervous system of patients with Leigh syndrome (LS) [7]. The genetic basis of LS is diverse and mutations causing this disease have been defined in many nDNA genes mainly associated with OXPHOS function, including $\mathrm{Cl}$ (NDUFV1, 2; NDUFS1, 2, 3, $4,7,8$; and NDUFA 1, 2, 9, 10, 12), CIII (UQCRQ), and CIV (NDUFA4) genes [8]. Nevertheless, LS also results from mtDNA mutations [8].

\section{mtDNA mutations}

Human SK-Hep1 hepatoma cells lacking mtDNA (rho ${ }^{\circ}$ cells) express more VEGF mRNA and protein than parental cells with $\mathrm{mtDNA}, \mathrm{rho}^{+}$cells. Moreover, conditioned medium from these rho ${ }^{\circ}$ cells increased the formation of tube-like structures from human umbilical vein endothelial cells and new blood vessels in chorioallantoic membrane assays [9]. Mice expressing a mutant mtDNA polymerase showed mtDNA depletion, reduced OXPHOS enzyme activities, and increased expression of VEGF mRNA [10].

Transmitochondrial cell lines, cytoplasmic hybrids, or cybrids can be used to confidently link a phenotype to mtDNA mutations. These cells share nDNA and differ in their mtDNA. By using this model, it was shown that cybrids obtained from mouse Lewis lung carcinoma cells, harboring a $\mathrm{Cl}$ mutation and having an OXPHOS defect, showed increased VEGF mRNA and protein levels and higher activity to induce neoangiogenesis than those with no mtDNA mutation [11,12].

In patients with mitochondrial myopathies attributable to heteroplasmic mtDNA mutations, in which mutated and wild-type mtDNA are present, muscle capillary density was twice that in sedentary healthy subjects and the capillary area was greatest in patients with the most severe muscle OXPHOS defects. It was also shown that, for each patient, capillary area around muscle fiber segments with defective OXPHOS function was more than twice that compared with muscle fiber segments with more preserved OXPHOS function. Thus, lower and higher OXPHOS function likely mirror a higher and lower percentage of mtDNA pathologic mutations, respectively [13].

In terms of visual disorders, an optic disc microangiopathy has been described as a typical ophthalmoscopic feature in patients who are asymptomatic for, or have, acute Leber hereditary optic neuropathy (LHON). LHON is an inherited form of vision loss that is caused by retinal ganglion cell dysfunction, mainly resulting from pathologic mutations in mtDNA genes. Interestingly, mtDNA J haplogroup increases the risk for LHON [14]. It was recently reported that, when peripapillary capillary vessel density was normalized by retinal nerve fiber layer thickness, the vascular network in the temporal sectors was increased in unaffected patients with LHON mutation. This suggests that the metabolic consequences of retinal ganglion cell mitochondrial impairment result in a compensatory vascular response, primarily affecting the area of the papillomacular bundle [15].

\section{OXPHOS inhibitors}

Given these earlier observations, mitochondrial dysfunction because of OXPHOS inhibitors might elevate VEGF levels. Supporting this assertion, in vitro pretreatment of human adiposederived stroma cells with $\mathrm{Cl}$ or $\mathrm{Clll}$ inhibitors (rotenone or antimycin, respectively) increased VEGF secretion. Following their injection into mice, the angiographic score and capillary density also increased [16]. Another $\mathrm{Cl}$ inhibitor (1-methyl-4-phenyl-1,2,3,6-tetrahydropyridine), frequently used to model Parkinson disease, also increased the number of VEGF-expressing neurons and of blood vessels in the substantia nigra of parkinsonian-rendered monkeys [17]. Treatment of human brain microvascular pericytes with the CIV inhibitor sodium cyanide also resulted in increased expression of VEGF [18]. Hydrogen sulfide, another CIV inhibitor, also has proangiogenic effects [19]. The ATP synthase inhibitor oligomycin increased VEGF protein production in the human U-937 monocytic cell line [20]. Likewise, 4-hydroxy-2-nonenal increased mtDNA point mutations and reduced CIII and CIV activity and oxygen consumption in primary rheumatoid arthritis synovial fibroblasts (RASF). This compound also increased RASF VEGF immunofluorescence staining and VEGF secretion. The number of tube-like structures produced by human umbilical vein endothelial cells was also increased by 4-hydroxy-2-nonenal-treated RASF-conditioned medium [21].

\section{Opposite observations}

The earlier results suggest that OXPHOS deficiency promotes pathological angiogenesis. However, some results point in an opposite direction. For example, inhibition of the nDNAencoded mitochondrial tryptophanyl-tRNA synthetase in endothelial cells reduced angiogenesis [22]; mutations in mtDNA-encoded tRNA genes decreased VEGF levels and angiogenesis [23,24]; inhibitors of mitochondrial translation (tigecycline and doxycycline) reduced neovascularization [25,26]; and rotenone and antimycin decreased VEGF production and angiogenesis in human ovarian cancer OVCAR-3, liver cancer HepG2 or mouse sarcoma-180 cells
$[27,28]$. Therefore, the relationship between pathological angiogenesis and OXPHOS function is complex and more studies are required for it to be fully understood.

\section{Pathological angiogenesis is a feature of many OXPHOS disorders}

Pathological angiogenesis can be observed in a range of disorders [29,30], such as ocular [31,32], rheumatic diseases [33,34], or cancer [35,36].

This might share a common early cause in these disorders. Thus, hypoxia appears to be the stimulus for retinal $[37,38]$, synovial $[39,40]$, or tumoral [41] angiogenesis. An essential adaptation to sustained hypoxia is the repression of mitochondrial respiration [42-44]. Angiogenesis frequently occurs in cancer, but nonangiogenic tumours can grow without triggering new vessel formation. Energy metabolism varies between angiogenic and nonangiogenic tumours. The latter show higher expression of proteins associated with OXPHOS and mitochondrial biogenesis $[45,46]$. It might be that OXPHOS dysfunction explains why pathological angiogenesis occurs in these diseases.

Primary OXPHOS disorders, such as those mentioned earlier, are not common [47]. However, many prevalent diseases are secondary OXPHOS disorders [48]. Mitochondrial alterations are often found in many of these angiogenesis-related disorders. Thus, retinal pigment epithelium from patients with AMD shows a decrease in the number and area of mitochondria compared with age-matched controls [49]. Mitochondrial oxygen consumption was significantly lower in retinal pigment epithelium from donors with AMD [50]. Mitochondrial number and COXIV protein levels were diminished in high glucose-cultured bovine retina endothelial cells. Moreover, in diabetic mouse retina or retina from patients with proliferative diabetic retinopathy, mtDNA was damaged and mtDNA copy number, mtDNA-encoded genes, mRNA amount, nDNA-encoded COXIV protein levels, and citrate synthase activity were all decreased [51]. In rheumatoid arthritis (RA), synovial fibroblasts undergo a shift in ATP generation from OXPHOS to glycolysis, which could be an adaptation to the joint microenvironment, similar to that seen in tumor cells $[40,52,53]$. Regarding cancer, mtDNA mutations are common and tumor growth is promoted by mtDNA mutations affecting OXPHOS function [54-56].

If an important OXPHOS dysfunction can increase pathological angiogenesis, then mtDNA haplogroup-associated OXPHOS variation might be a susceptibility and/or resistance factor for 
aspects of these angiogenesis-related diseases. For example, as discussed earlier, OXPHOS capability was lower in cybrids from the JT haplogroup [57] and OXPHOS electron transport rate was higher in cybrids from the $U$ haplogroup [58]. Interestingly, the JT haplogroup appears to be a susceptibility factor for nAMD [59], and individuals from this haplogroup showed a worse response to nAMD intravitreal ranibizumab therapy [3]. By contrast, the $U$ haplogroup appears to be a resistance factor for proliferative diabetic retinopathy $[60,61]$, and individuals from this haplogroup showed a better response to nAMD intravitreal ranibizumab therapy [3]. In RA, radiographic erosions were significantly associated with the JT haplogroup [62] and, in early RA, an association was reported between erosions and increased intraarticular blood flow [63]. The mtDNA haplogroup has also been related to cancer risk [6466].

Thus, these results suggest that OXPHOS function is impaired, and should be analyzed, in other angiogenesis-related disorders [29].

\section{Enhancing OXPHOS function would reduce pathological angiogenesis}

If mechanisms underlying angiogenesis-dependent diseases are related, therapies for one disease might be helpful for others. OXPHOS dysfunction appears to contribute to these pathologies. Therefore, interventions to improve OXPHOS function would have widespread clinical applications. It was recently reported that the loss of $\beta$-adrenergic receptor signaling leads to inhibition of angiogenesis through enhancement of endothelial OXPHOS [67]. Also, the loss of the glycolytic isoenzyme pyruvate kinase $M 2$ increased oxygen consumption rate and led to angiogenic sprouting defects [68]. Two main types of antiangiogenic drug have been developed: (i) monoclonal antibodies, or related molecules, targeting either VEGF or the extracellular domain of its receptor; and (ii) tyrosine-kinase inhibitors (TKI), targeting the intracellular kinase domains of VEGF, or other tyrosine kinase receptors. It was recently shown that monoclonal antibody-related molecules increased the respiration rate in human adult retinal pigment epithelial-19 (ARPE-19) cells [69]. Moreover, it was also shown that tumor metabolism was reprogrammed in response to TKI, with increased mitochondrial metabolism. Thus, TKI-treated tumor tissue had intense Cll staining and its respiratory capacity was higher than that of control-treated tumor tissue [70].

Several agents have already been proposed that, by acting on different cell targets, increase mitochondrial biogenesis [14]. Some of these compounds also appear to be beneficial for angiogenesis-related diseases. For example,caffeine [71,72]; polyphenols, such as curcumin, epigallocatechin gallate, quercetin, and resveratrol [73-76]; thiazolidinediones, such as rosiglitazone $[77,78]$; valproic acid $[79,80] ; \Delta^{9}$ tetrahydrocannabinol [81,82]; and $\omega 3$-polyunsaturated fatty acids, such as docosahexaenoic or eicosapentaenoic acids [83]. These compounds could be used, along with other antiangiogenic drugs, as adjuvants for either all patients with angiogenesis-related diseases or those belonging to a particular mtDNA haplogroup with a high genetic risk.

\section{Concluding remarks}

This retrospective analysis reveals a previously unrecognized pattern, that OXPHOS dysfunction might be an etiological factor for pathological angiogenesis. These observations lead to a new hypothesis whereby OXPHOS inducers would be interesting candidate drugs for the treatment of diseases involving OXPHOS dysfunction. Nevertheless, angiogenesis-related diseases also include those in which angiogenesis is hampered [29]. In such cases, OXPHOS inhibitors might act as proangiogenic drugs.

\section{Acknowledgments}

This work was supported by grants from the Instituto de Salud Carlos III (ISCIII) (PI17/00021 and PI17/00166), Departamento de Ciencia,

Tecnología y Universidad del Gobierno de Aragón (Grupos de Referencia B33_17R), and the FEDER Funding Program from the European Union. CIBERER is an initiative of the ISCIII. Sponsors did not have any role in the study design; in the collection, analysis, and interpretation of data; in writing of the report; and in the decision to submit the paper for publication.

\section{References}

1 Mitchell, P. et al. (2018) Age-related macular degeneration. Lancet 392, 1147-1159

2 Gerding, H. (2014) Ranibizumab treatment in agerelated macular degeneration: a meta-analysis of oneyear results. Klin. Monbl. Augenheilkd. 231, 427-431

3 Esteban, O. et al. (2019) Effect of mitochondrial haplogroups on ranibizumab response in neovascular age-related macular degeneration patients: a pilot study. Acta Ophthalmol. 97, e133-e134

4 Gimenez-Roqueplo, A.P. et al. (2001) The R22X mutation of the SDHD gene in hereditary paraganglioma abolishes the enzymatic activity of complex II in the mitochondrial respiratory chain and activates the hypoxia pathway. Am. J. Hum. Genet. 69, 1186-1197

5 Gimenez-Roqueplo, A.P. et al. (2002) Functional consequences of a SDHB gene mutation in an apparently sporadic pheochromocytoma. J. Clin. Endocrinol. Metab 87, 4771-4774

6 Chang, J. et al. (2014) A mutation in the mitochondrial protein UQCRB promotes angiogenesis through the generation of mitochondrial reactive oxygen species. Biochem. Biophys. Res. Commun. 4554, 290-297

7 Lake, N.J. et al. (2015) Leigh syndrome: neuropathology and pathogenesis. J. Neuropathol. Exp. Neurol. 74, 482-492

8 Lake, N.J. et al. (2016) Leigh syndrome: one disorder, more than 75 monogenic causes. Ann. Neurol. 79, 190 203

9 Cheon, H. et al. (2010) Loss of mitochondrial DNA enhances angiogenic and invasive potential of hepatoma cells. Oncol. Rep. 23, 779-786

10 Singh, B. et al. (2018) Reversing wrinkled skin and hair loss in mice by restoring mitochondrial function. Cell Death Dis. 9, 735

11 Koshikawa, N. et al. (2003) Constitutive upregulation of hypoxia-inducible factor-1alpha mRNA occurring in highly metastatic lung carcinoma cells leads to vascular endothelial growth factor overexpression upon hypoxic exposure. Oncogene 22, 6717-6724

12 Ishikawa, K. et al. (2008) ROS-generating mitochondrial DNA mutations can regulate tumor cell metastasis. Science 320, 661-664

13 Taivassalo, T. et al. (2012) Increased capillaries in mitochondrial myopathy: implications for the regulation of oxygen delivery. Brain 135, 53-61

14 Ruiz-Pesini, E. et al. (2018) Increasing mtDNA levels as therapy for mitochondrial optic neuropathies. Drug Discov. Today 23, 493-498

15 Balducci, N. et al. (2018) Peripapillary vessel density changes in Leber's hereditary optic neuropathy: a new biomarker. Clin. Exp. Ophthalmol. 46, 1055-1062

16 Carriere, A. et al. (2009) Preconditioning by mitochondrial reactive oxygen species improves the proangiogenic potential of adipose-derived cellsbased therapy. Arterioscler. Thromb. Vasc. Biol. 29, 1093-1099

17 Barcia, C. et al. (2005) Changes in vascularization in substantia nigra pars compacta of monkeys rendered parkinsonian. J. Neural Transm. 112, 1237-1248

18 Bai, Y. et al. (2015) Pericytes contribute to the disruption of the cerebral endothelial barrier via increasing VEGF expression: implications for stroke. PLoS One 10, e0124362

19 Longchamp, A. et al. (2018) Amino acid restriction triggers angiogenesis via GCN2/ATF4 regulation of VEGF and H2S production. Cell 173, 117-129

20 Satake, S. et al. (1998) Up-regulation of vascular endothelial growth factor in response to glucose deprivation. Biol. Cell 90, 161-168

21 Balogh, E. et al. (2018) Oxidative stress impairs energy metabolism in primary cells and synovial tissue of patients with rheumatoid arthritis. Arthritis Res. Ther. 20, 95

22 Wang, M. et al. (2016) Wars2 is a determinant of angiogenesis. Nat. Commun. 7, 12061

23 Chang, J.C. et al. (2017) Peptide-mediated delivery of donor mitochondria improves mitochondrial function and cell viability in human cybrid cells with the MELAS A3243G mutation. Sci. Rep. 7, 10710

$24 \mathrm{Jia}$, Z. et al. (2019) A coronary artery disease-associated tRNAThr mutation altered mitochondrial function, apoptosis and angiogenesis. Nucleic Acids Res. 47, 2056-2074

25 Samtani, S. et al. (2009) Doxycycline-mediated inhibition of choroidal neovascularization. Invest. Ophthalmol. Vis Sci. 50, 5098-5106 
26 Xiong, Y. et al. (2018) Tigecycline as a dual inhibitor of retinoblastoma and angiogenesis via inducing mitochondrial dysfunctions and oxidative damage. Sci. Rep. 8, 11747

27 Maeda, M. et al. (2006) Inhibition of angiogenesis and HIF-1alpha activity by antimycin A1. Biol. Pharm. Bull. 29, 1344-1348

28 Xia, C. et al. (2007) Reactive oxygen species regulate angiogenesis and tumor growth through vascular endothelial growth factor. Cancer Res. 67, 10823-10830

29 Carmeliet, P. (2003) Angiogenesis in health and disease. Nat. Med. 9, 653-660

30 Folkman, J. (2007) Angiogenesis: an organizing principle for drug discovery? Nat. Rev. Drug Discov. 6, 273-286

31 Witmer, A.N. et al. (2003) Vascular endothelial growth factors and angiogenesis in eye disease. Prog. Retin. Eye Res. 22, 1-29

32 Paulus, Y.M. and Sodhi, A. (2017) Anti-angiogenic therapy for retinal disease. Handb. Exp. Pharmacol. 242, 271-307

33 Marrelli, A. et al. (2011) Angiogenesis in rheumatoid arthritis: a disease specific process or a common response to chronic inflammation? Autoimmun. Rev. 10, 595-598

34 Tas, S.W. et al. (2016) Targeting of proangiogenic signalling pathways in chronic inflammation. Nat. Rev. Rheumatol. 12, 111-122

35 Carmeliet, P. and Jain, R.K. (2000) Angiogenesis in cancer and other diseases. Nature 407, 249-257

36 Weis, S.M. and Cheresh, D.A. (2011) Tumor angiogenesis: molecular pathways and therapeutic targets. Nat. Med. 17, 1359-1370

37 Sapieha, P. et al. (2010) Proliferative retinopathies: angiogenesis that blinds. Int. J. Biochem. Cell Biol. 42, 512

38 Kent, D.L. (2014) Age-related macular degeneration beyond anti-angiogenesis. Mol. Vis. 20, 46-55

39 Konisti, S. et al. (2012) Hypoxia-a key regulator of angiogenesis and inflammation in rheumatoid arthritis. Nat. Rev. Rheumatol. 8, 153-162

40 Fearon, U. et al. (2016) Hypoxia, mitochondrial dysfunction and synovial invasiveness in rheumatoid arthritis. Nat. Rev. Rheumatol. 12, 385-397

41 Gatenby, R.A. and Gillies, R.J. (2004) Why do cancers have high aerobic glycolysis? Nat. Rev. Cancer 4, $891-$ 899

42 Papandreou, I. et al. (2006) HIF-1 mediates adaptation to hypoxia by actively downregulating mitochondria oxygen consumption. Cell Metab. 3, 187-197

43 Zhang, H. et al. (2007) HIF-1 inhibits mitochondrial biogenesis and cellular respiration in VHL-deficient renal cell carcinoma by repression of C-MYC activity. Cancer Cell 11, 407-420

44 Denko, N.C. (2008) Hypoxia, HIF1 and glucose metabolism in the solid tumour. Nat. Rev. Cancer 8 705-713

$45 \mathrm{Hu}$, J. et al. (2005) Gene expression signature for angiogenic and nonangiogenic non-small-cell lung cancer. Oncogene 24, 1212-1219

46 Donnem, T. et al. (2018) Non-angiogenic tumours and their influence on cancer biology. Nat. Rev. Cancer 18 323-336

47 Gorman, G.S. et al. (2016) Mitochondrial diseases. Nat Rev. Dis. Primers 2, 16080

48 Wallace, D.C. (2013) A mitochondrial bioenergetic etiology of disease. J. Clin. Invest. 123, 1405-1412
49 Feher, J. et al. (2006) Mitochondrial alterations of retina pigment epithelium in age-related macula degeneration. Neurobiol. Aging 27, 983-993

50 Ferrington, D.A. et al. (2017) Altered bioenergetics and enhanced resistance to oxidative stress in human retinal pigment epithelial cells from donors with agerelated macular degeneration. Redox Biol. 13, 255-265

51 Santos, J.M. et al. (2011) Mitochondrial biogenesis and the development of diabetic retinopathy. Free Radic. Biol. Med. 51, 1849-1860

52 Garcia-Carbonell, R. et al. (2016) Critical role of glucose metabolism in rheumatoid arthritis fibroblast-like synoviocytes. Arthritis Rheumatol. 68, 1614-1626

53 Kim, E.K. et al. (2017) IL-17-mediated mitochondrial dysfunction impairs apoptosis in rheumatoid arthritis synovial fibroblasts through activation of autophagy. Cell Death Dis. 8, e2565

54 Petros, J.A. et al. (2005) mtDNA mutations increase tumorigenicity in prostate cancer. Proc. Natl. Acad. Sci. U. S. A. 102, 719-724

55 Wallace, D.C. (2012) Mitochondria and cancer. Nat. Rev Cancer 12, 685-698

56 Cruz-Bermudez, A. et al. (2017) Spotlight on the relevance of mtDNA in cancer. Clin. Transl. Oncol. 19, 409-418

57 Gomez-Duran, A. et al. (2012) Oxidative phosphorylation differences between mitochondrial DNA haplogroups modify the risk of Leber's hereditary optic neuropathy. Biochim. Biophys. Acta 1822, 1216 1222

58 Gomez-Duran, A. et al. (2010) Unmasking the causes of multifactorial disorders: OXPHOS differences between mitochondrial haplogroups. Hum. Mol. Genet. 19, 3343-3353

59 Mueller, E.E. et al. (2012) Mitochondrial haplogroups and control region polymorphisms in age-related macular degeneration: a case-control study. PLoS One 7, e30874

60 Estopinal, C.B. et al. (2014) Mitochondrial haplogroups are associated with severity of diabetic retinopathy. Invest. Ophthalmol. Vis. Sci. 55, 5589-5595

61 Martikainen, M.H. et al. (2015) Association of mitochondrial DNA haplogroups and vascular complications of diabetes mellitus: a population-based study. Diab. Vasc. Dis. Res. 12, 302-304

62 Duhn, P.H. et al. (2017) Mitochondrial haplogroups in patients with rheumatoid arthritis: No association with disease and disease manifestations. PLoS One 12, e0188492

63 Taylor, P.C. (2002) VEGF and imaging of vessels in rheumatoid arthritis. Arthritis Res. 4, S99-107

64 Bayona-Bafaluy, M.P. et al. (2011) Maternally inherited susceptibility to cancer. Biochim. Biophys. Acta 1807 , 643-649

65 Brinker, A.E. et al. (2017) Mitochondrial haplotype alters mammary cancer tumorigenicity and metastasis in an oncogenic driver-dependent manner. Cancer Res. 77, 6941-6949

66 Bussard, K.M. and Siracusa, L.D. (2017) Understanding mitochondrial polymorphisms in cancer. Cancer Res. 77, 6051-6059

67 Zahalka, A.H. et al. (2017) Adrenergic nerves activate an angio-metabolic switch in prostate cancer. Science 358, 321-326

68 Stone, O.A. et al. (2018) Loss of pyruvate kinase M2 limits growth and triggers innate immune signaling in endothelial cells. Nat. Commun. 9,4077
69 Sheu, S.J. et al. (2015) Differential effects of bevacizumab, ranibizumab and aflibercept on cell viability, phagocytosis and mitochondrial bioenergetics of retinal pigment epithelial cell. Acta Ophthalmol. 93, e631-e643

70 Navarro, P. et al. (2016) Targeting tumor mitochondrial metabolism overcomes resistance to antiangiogenics. Cell Rep. 15, 2705-2718

[71] Chen, J.F. et al. (2017) Adenosine receptors and caffeine in retinopathy of prematurity. Mol. Aspects Med. 55, 118-125

72 Tej, G. and Nayak, P.K. (2018) Mechanistic considerations in chemotherapeutic activity of caffeine. Biomed. Pharmacother. 105, 312-319

73 Yang, C.S. et al. (2009) Cancer prevention by tea: animal studies, molecular mechanisms and human relevance. Nat. Rev. Cancer 9, 429-439

74 Sulaiman, R.S. et al. (2014) Natural product inhibitors of ocular angiogenesis. Exp. Eye Res. 129, 161-271

75 Wang, Z. et al. (2015) Broad targeting of angiogenesis for cancer prevention and therapy. Semin Cancer Biol. 35, S224-S243

76 Ribeiro, A. et al. (2018) Antiangiogenic compounds: well-established drugs versus emerging natural molecules. Cancer Lett. 415, 86-105

77 Panigrahy, D. et al. (2003) Therapeutic potential of thiazolidinediones as anticancer agents. Expert Opin. Investig. Drugs 12, 1925-1937

78 Shen, L.Q. et al. (2008) Rosiglitazone and delayed onset of proliferative diabetic retinopathy. Arch. Ophthalmol. 126, 793-799

79 Blaheta, R.A. et al. (2005) Evolving anticancer drug valproic acid: insights into the mechanism and clinical studies. Med. Res. Rev. 25, 383-397

80 Berendsen, S. et al. (2012) Valproic acid for the treatment of malignant gliomas: review of the preclinical rationale and published clinical results. Expert Opin. Investig. Drugs 21, 1391-1415

81 Velasco, G. et al. (2007) Cannabinoids and gliomas. Mol. Neurobiol. 36, 60-67

82 Sledzinski, P. et al. (2018) The current state and future perspectives of cannabinoids in cancer biology. Cancer Med. 7, 765-775

83 Spencer, L. et al. (2009) The effect of omega-3 FAs on tumour angiogenesis and their therapeutic potential. Eur. J. Cancer 45, 2077-2086

\section{M.Pilar Bayona-Bafaluy 1,2,3,* \\ Olivia Esteban 2,4 \\ Javier Ascaso ${ }^{2,4}$ \\ Julio Montoya ${ }^{1,2,3}$ \\ Eduardo Ruiz-Pesini ${ }^{1,2,3,5, *}$}

${ }^{1}$ Departamento de Bioquímica, Biología Molecular y Celular, Universidad de Zaragoza, Zaragoza, Spain

${ }^{2}$ Aragón Health Research Institute (IIS Aragón), Zaragoza, Spain

${ }^{3}$ Centro de Investigaciones Biomédicas en Red de Enfermedades Raras (CIBERER), SpainQ3

${ }^{4}$ Department of Ophthalmology, Hospital Clínico Q3 Universitario 'Lozano Blesa', Zaragoza, Spain

${ }^{5}$ Fundación ARAID, Zaragoza, Spain

*Corresponding authors. 